\title{
DRIVING PERFORMANCE OF DRUG-IMPAIRED BUS DRIVERS IN WORK ZONE AREAS
}

\author{
Chris Deits ${ }^{1}$, Linda Ng Boyle ${ }^{1}$, \& John Morrison ${ }^{2}$ \\ ${ }^{1}$ Department of Industrial and Systems Engineering, University of Washington, \\ Seattle, Washington, USA \\ ${ }^{2}$ Cahill Swift, LLC, Boston, Massachusetts, USA \\ E-mail: linda@uw.edu
}

\begin{abstract}
Summary: There are several safety issues in work zone areas due to decrease lane width, and presence of large equipment and on-site workers. The safety issues are compounded for drivers that are also drug impaired. In this study, we examine the driver performance of 18 commercial bus operators in a simulated environment while they are under the influence of a benzodiazepine drug (Triazolam). The findings show that those drivers under the influence of the drug had higher steering entropy and greater difficulty staying close to the intended travel lane when compared to those who were not under the influence of the drug. These wider travelling distances around work barrels, by those under the influence of Triazolam, could have a potential impact on the safety of nearby construction workers and heavy machine operators.
\end{abstract}

\section{INTRODUCTION}

According to Khattak et al (2002), there are approximately 700 traffic-related fatalities, and over 75,000 injury and non-injury crashes per year in work zone areas in the US. The number of crashes in work zone areas are attributed to several factors, including the size of vehicle passing through the work area (Khattak and Targa, 2004), work zone configuration, speed limits through the zones, and environmental conditions (Li and Bai, 2008 and Harb et al., 2008). Studies using driving simulators are quite appropriate in examining issues related to work zones without increasing traffic congestion or decreasing safety (e.g, Morgan and Hancock, 2010, and Bella, 2005).

Work zone safety can also be impacted by drivers under the influence of drugs, whether prescription or illegal. Specific to prescription drug use, one type of drug widely used by drivers is short-acting benzodiazepines or non-benzodiazepine hypnotic drugs (i.e. sleep aids, sleeping pills) (van Laar and Volkerts, 1998). Based on data from the large truck crash causation study (US DOT, 2010), benzodiazepines were the second most used psychoactive drug among crashinvolved commercial drivers (with antidepressants being the first). One particular drug that has been used in the past is Triazolam (Halcion). Triazolam is a short acting benzodiazepine that has been used in patients who have difficulty in falling asleep (Roth et al., 1980) and in dental medicine to calm fearful patients (Milgram et al., 1994). This drug has been shown to increase crash risk by 13 fold during the first week of new prescription use when compared to those drivers not taking triazolam (Neutel, 1995).

This current study examines the implications of this drug on driver performance as bus drivers traverse over a simulated work zone area. We hypothesize that drivers who have higher dosage 
of Triazolam will have more difficult time traversing around a work zone area. The data used for this study comes from a larger study aimed at developing technology for measuring the impact of psychoactive prescription medications on the same-day and next-day driving performance of actively employed bus operators that hold a commercial driver license (CDL).

\section{METHODS}

\section{Participants}

The participants were employed as a transit, coach or school bus driver around Iowa City, Iowa and recruited through newspaper ads, flyers at driving facilities, and through presentations as requested. Each participant completed three sessions over a 3-week period. Participants were excluded if they were currently using any other drugs known to enhance the effects of Triazolam (including grapefruit juice and birth control pills). Each session spanned 2 days (dosed and dayafter drives). There was also a day each of training, orientation, and physical examination. Drivers were also debriefed with a phone call that lasted approximately 1 hour. Hence, they were compensated $\$ 640$ if they completed all drivers and tests.

There were a total of 28 participants in the study but only 18 were used in this analysis because several participants did not complete all three dose trials. For ethical reasons, some drivers were removed from the study because their dosage level remained high on the day-after saliva specimen.

\section{Equipment}

A modern, high-fidelity bus driving simulator built by FAAC, Inc. and owned by the Paducah Area Transit System (PATS) was leased for this study. The FAAC bus is built on a Gillig Bus front end and has a 360-degree display through seven video-channels (3 front video projectors, 2 side displays, 2 rear displays) and a complete set of bus controls. The simulator is housed in a large climate-controlled truck trailer purpose-build for the simulator with a $300 \mathrm{KV}$ diesel electric generator for regulated electrical power for the simulator and computers. The simulator was driven from Paducah KY to Iowa City and parked in the parking lot of the National Advanced Driving Simulator (NADS) for the two-month duration of the experimental period.

\section{Procedure}

There were three experimental sessions spaced at least a week apart. Each experimental session consisted of five simulator drives ( 10 minutes each) over a two-day period: four drives on day one and one drive the very next day. The drives on the first day of each session were 40 minutes apart and designed to measure current impairment through the known peak effect of the drug at approximately 90 minutes. The capsule for the day was ingested immediately following the completion of the first drive of the day, to provide a pre-dose measure of driver performance. Participants returned the next day to conduct the fifth drive of the session, to measure any carryover effects of the drug after a period of 10-14 hours including sleep. The next-day drive was a repetition of the pre-dose drive and no capsules were ingested. Each driver completed three 
sessions of five drives. The analyses presented in this paper are focused only on those scenarios with a work-zone (i.e., $3^{\text {rd }}$ and $4^{\text {th }}$ drives on day 1 ).

Subjects completed a short psychomotor test battery immediately before the first simulator drives on each test day. The battery was a subset of the ANAM (Automated Neuropsychological Assessment Metrics) (http://c-shop.ou.edu/anam4.htm). Psychomotor tests included a mood and sleepiness scale and six tests to measure reaction time, choice behavior, rotary tracking, math skills and short-term memory. The experimental protocol also included questionnaires after each session. Additionally, saliva samples were collected from each participant immediately following each experimental drive. The saliva samples were analyzed for Triazolam content to provide a surrogate measure for the instantaneous plazma levels of the active drug.

As stated earlier, this study examined the work zone sections only which included two-lanes (or one-lane in each direction) and five barrels in a staggered position that purposely blocked the driver's lane of travel, forcing drivers to maneuver around them. It is noted that due to constraints with the simulator, the work zone configurations were not designed to federal guidelines. The three work zone scenarios differed by the placement of the barrel and the length of the road segment. Scenario 1 also included a stop sign immediately preceding the work zone area (470 meters long). Scenario 2 was a straight road and drivers could see the barrels (376 m). Scenario 3 included a right hand turn immediately preceding the work zone area $(792 \mathrm{~m})$. Participants performed the experiment starting midday to late evening, with the majority of studies conducted in the afternoon due to driver work schedules.

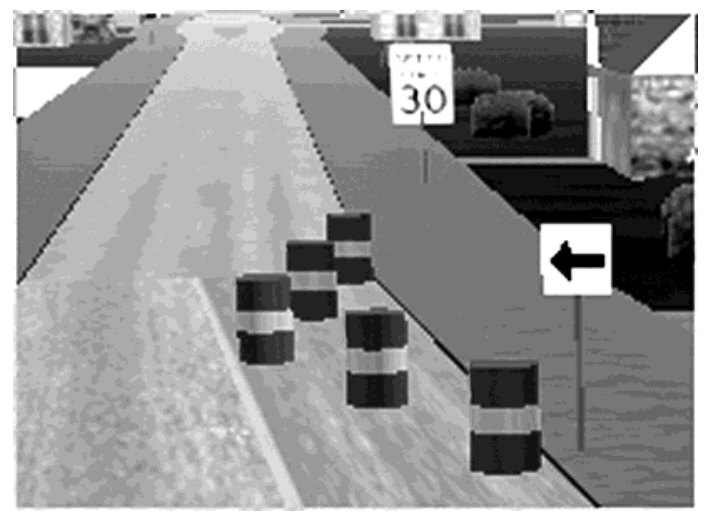

Figure 1. Example of barrel configuration

\section{Experimental design}

Triazolam (Halcion) was administered in a placebo-controlled, double-blind, randomized crossover design. On intake, each participant was randomized into one of the six groups representing the possible combinations of the experimental doses, placebo, 0.125 and 0.250 , administered in a randomized order. The dose levels chosen are the most common dose levels prescribed for those suffering from sleeping problems. Each participant, over the three-week study period, received the $0.125 \mathrm{mg}$ dose of Triazolam, the $0.250 \mathrm{mg}$, and the placebo dose in double-blind a randomized order. The sucrose placebo tablet, and the 0.125 and $0.250 \mathrm{mg}$ capsules were administered in a standard drug capsule filled with sucrose to mask taste. 


\section{Dependent Measures}

The two dependent measures examined included the total driving distance of the driver's path around the barrels and steering entropy. Total driving distance was examined as a surrogate for vehicle control. Participants that were cognitively impaired by Triazolam may not be able to control their vehicle with precision and any exaggerated lateral movement may equate to a longer distance traveled.

Steering entropy is a measure that has been used to examine increased mental workload (Nakayama, 1999). A related measure based on the entropy calculation is the $90 \%$ interquartile range, $\gamma$, since entropy is centered normally distributed with mean=zero and standard deviation $=\sigma$. This measure $(\gamma)$ will give similar insights, compared to the traditional steering entropy, for drivers when a placebo baseline is not available across all drivers as is the case in this analysis.

\section{Explanatory Variables}

The dependent measure of interest was dose level and included three levels: placebo, $0.125 \mathrm{mg}$ dose, and $0.250 \mathrm{mg}$ dose of Triazolam. Covariates were also included given the potential that they may impact the outcome. These include the score for the simulator sickness questionnaire (SSQ) and the interaction between the SSQ score and the Triazolam saliva concentration, and dose administration order. A continuous variable for BMI score and the interaction of BMI with Triazolam Saliva concentration was also included as a covariate since studies show that those with larger body mass may have increased sensitivity to benzodiazepines which includes Triazolam (Greenblatt et. al, 1984 and Derry et al. 1995).

The number of years as a commercial driver and the driver's age were also considered as covariates. However, a moderately strong correlation existed between these variables and the BMI score so they were not included to avoid issues with multicollinearity (Farrar and Glauber, 1967).

\section{RESULTS}

The 18 participants had a mean age of 24.56 years $(\mathrm{sd}=6.48)$ and mean commercial driving experience of 2.28 years ( $s d=1.90)$. There were 17 males and only one female. Due to this gender disparity, gender effects are not examined. The mean body mass index (BMI) of these participants was 26.72 (sd=6.24) with a range of 20 (considered normal) to 42 (considered obese). With three different driving scenarios and 18 participants, there was a total of 54 individual drives examined in this analysis.

Each participant had three observations representing each of the three work zone scenarios. Linear mixed effects models were used to estimate the possible effects of Triazolam on the participants' total driving distance through the work zones and differences in steering entropy. This type of model allows for repeated observations to be taken into account. This model was fitted using the statistical package R (2.10.1) and the "nlme" package within that software. 
A random intercept was estimated to account for the random effects unique to each driver. After fitting the model and observing the residuals vs. fitted values, the variable, driving distance, was also transformed by the log function to remove a slight mean-variance relationship in the residuals.

\section{Distance Travelled}

There was a significant difference between those who had received the placebo and those who did not $(\mathrm{F}(2,28)=5.09, \mathrm{p}=0.01)$. More specifically, those who had the $0.125 \mathrm{mg}$ dose of Triazolam, $(\mathrm{t}(28)=2.87)$ travelled further distances than those who had received the placebo. Those with the $0.250 \mathrm{mg}$ dose $(\mathrm{t}(28)=-1.01, \mathrm{p}>0.2)$ did not significantly differ from those who had the placebo. While those participants who received the $0.25 \mathrm{mg}$ dose did not, on average, significantly differ from those who had received the placebo, the variability over the distance traveled for the participants who received the $0.250 \mathrm{mg}$ was 1.5 times greater when compared to those who had received the placebo (see Figure 2 for an example).

There was a difference in the physical configuration of each work zone area and this did impact the distance travelled $(\mathrm{F}(2,28)=27400, \mathrm{p}<0.001)$. Hence, a covariate was included to account for this effect. Dose order also impacted the distance traversed $(F(5,11)=3.29, p=0.046)$ and was also included in the model.

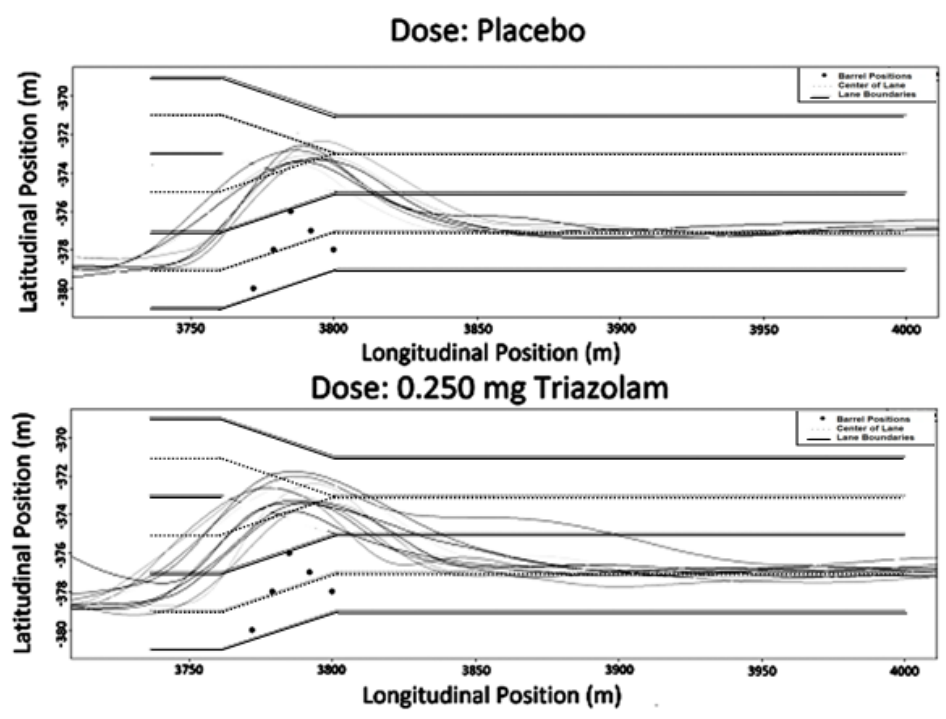

Figure 2. Driver's paths under Placebo (top) and 0.250 (bottom) dosage (First Session, Third Drive)

\section{Steering Entropy}

Drivers with the 0.25 dose had an increase in steering entropy $(F(2,28)=6.67, p=.004)$. The model was also adjusted for differences in the drive scenarios given that there was a higher steering entropy for the 2nd scenario (the short straight road) when compared to the $1^{\text {st }}$ and 3rd drives. Those drivers with a higher BMI also had significantly lower steering entropy $(F(1,11)=8.19, \mathrm{p}<0.05)$. There were no other significant differences observed. 


\section{DISCUSSION}

Studies show that benzodiazepines impact psychomotor skills (Mintzer, 2002; Rush et al, 1993) and ultimately driving performance (Bramness et al., 2002). We had hypothesize that drivers who have higher dosage of Triazolam will have more difficult time traversing around a work zone area. Based on the results of the present study, drivers with 0.125 dose did travel further around the barrels than the drivers with the placebo, but interestingly, no difference in travel distance was observed for those with 0.25 . Our data analysis examined the impact of dosage level within specific scenarios, but oftentimes, changes in behavior need to be observed over time. And drivers with $0.250 \mathrm{mg}$ dose did show increased variability in their behavior while navigating the work zone area. This result and the significant increase in steering entropy observed under this treatment level are indications that there may be some cognitive impairment due to the Triazolam.

Drivers under the influence may drive further away from the barrels than those who had a placebo because they are cautious (McMillan et al, 1989). However, they could also be having a more difficult time maintaining an efficient path around the barrels. Other studies do show greater variations in lane position for those drivers that are cognitively impaired (Ramaekers et al, 2000). Drivers who drive too far away from the barrels may also have a greater likelihood of colliding with ongoing construction. The increased demand associated with operating the vehicle while drug-impaired could potentially limit their ability to attend to other safety critical situations.

However, the results do need to be interpreted cautiously as there were several study limitations. There was difficulty in retaining participants for the full study (3 weeks) and the power of the study was quite low. There were also very diverse drive scenarios (in terms of length and configurations) that prohibited a completely crossed design. The analysis did control for many variables statistically but it would have been beneficial to control for these experimentally. It is important to recognize that this was a proof of concept study only. The data for this analysis is part of a large study to assess safe driving performance under the influence of Triazolam. This specific study focused on drive performance in work zone areas but clearly, more research will be needed to identify specific scenarios where those drivers under the influence are at the greatest risk.

\section{ACKNOWLEDGMENTS}

This work described in this paper was sponsored by the US DOT - Federal Transit Administration. The authors acknowledge faculty and staff at the U. Iowa who worked diligently on IRB issues, data collection and reduction for this study (O. Ahmad, D. McGehee, G. Milavetz, M. Reyes, S. Salisbury). 


\section{REFERENCES}

Bella, F. (2005) Validation of a driving simulator for work zone designs. Transportation Research Record: Journal of the Transportation Research Board, 1937, 136-144

Bramness et al. (2002), Clinical impairment of benzodiazepines_relation between benzodiazepine concentrations and impairment in apprehended drivers. Drug and Alcohol Dependence, 68, 1 131-141

Derry, C. et al. (1995), Pharmacokinetics and Pharmacodynamics of Triazolam After Two Intermittent Doses in Obese and Normal-Weight Men, Journal of Clinical Psychopharmacology,15, 3, 197-205

Farrar, D. and Glauber, R. (1967), Multicollinearity in Regression Analysis: The Problem Revisited, The Review of Economics and Statistics, 49, 1, 92-107

Greenblatt, D. J. et al. (1984), Effect of Age, Gender, and Obesity on Midazolam Kinetics, Anesthesiology, 61, 27-37

Khattak et al (2002) effects of work zone presence on injury and non-injury crashes, Accident Analysis \& Prevention, 34, 1, 19-29

McMillen, D., Smith, S., and Wells-Parker (1989) The effects of alcohol, expectancy, and sensation seeking on driving risk taking, Addictive Behaviors, 14,1, 447-483

Milgram P. et al. (1994) The efficacy and memory effects of oral triazolam premedication in highly anxious dental patients, anesthesia progress, 41, 3, 70-76

Mintzer, M.Z. (2002) Alcohol and Triazolam: differential effects on memory, psychomotor performance and subjective ratings of effects, Behavioral Pharmacology, 13, 8, 653-658

Nakayama et al. (1999) Development of a steering entropy method for evaluating driver workload, Paper presented at SAE International Congress and Exposition, Detroit, MI.

Morgan, J., Duley, A.R. and Hancock, P.A. (2010) Driver responses to differing urban work zone configurations, Accident Analysis \& Prevention, 42, 3, 978-985

Neutal, C.I. (1995) Risk of traffic accident after a prescription from a benzodiapine. American Epidemiology, 5, 3, 239-244.

Ramaekers, J.G. et al. (2000) Marijuana, alcohol and actual driving performance, Human Psychopharmacology: Clinical and Experimental, 15,7,551-558.

Roth et al (1980) The effects of flurazepam, lorazepam, and triazolam on sleep and memory, Psychopharmacology, 70, 231-237

Rush et al. (1993) The acute effects of Triazolam and Lorazepam on Human Learning, Performance and Subject Ratings, Journal of Pharmacology and Experimental Therapeutics, 264, 3, $1218-1226$

US DOT (2010) Federal Motor Carrier Safety Adminstration - Large Truck Crash Causation Study, accessed on Nov 20, 2010 from http://www nass.nhtsa.dot.gov/ltccs_pub/searchform.aspx.

van Laar, M.W. and Volkerts, E.R. (1998) Driving and benzodiazepine use - evidence that they do not mix, CNS Drugs, 14, 5, 383-396. 